\title{
Assessment of Angular Spectral Distributions of Laser Accelerated Particles for Simulation of Radiation Dose Map in Target Normal Sheath Acceleration Regime of High Power Laser-Thin Solid Target Interaction-Comparison with Experiments
}

\author{
Andreea Groza ${ }^{1}$, Alecsandru Chirosca ${ }^{2, *}$, Elena Stancu ${ }^{3}{ }^{-}$, Bogdan Butoi ${ }^{1}$, Mihai Serbanescu ${ }^{4,5}$, \\ Dragana B. Dreghici ${ }^{1,2}$ and Mihai Ganciu ${ }^{1}$ \\ 1 Low Temperature Plasma Department, National Institute for Laser, Plasma and Radiation Physics (INFLPR), \\ Atomistilor Str. No. 409, 077125 Magurele, Ilfov County, Romania; andreea.groza@inflpr.ro (A.G.); \\ ro_medeus@yahoo.com (B.B.); dragana.dreghici@inflpr.ro (D.B.D.); mihai.ganciu@inflpr.ro (M.G.) \\ 2 Nuclear Physics Department, Faculty of Physics, University of Bucharest, 077125 Magurele, Romania \\ 3 STARDOOR Department, National Institute for Laser, Plasma and Radiation Physics, Atomistilor Str. \\ No. 409, 077125 Magurele, Romania; elena.stancu@inflpr.ro \\ 4 Centre for Advanced Laser Technology (CETAL-PW), National Institute for Laser, Plasma and \\ Radiation Physics, Atomistilor Str. No. 409, 077125 Magurele, Ilfov County, Romania; \\ mihai.serbanescu@inflpr.ro \\ 5 Faculty of Electronics, Telecommunications and Information Technology, Politehnica University \\ of Bucharest (UPB), Splaiul Independentei 313, 060042 Bucharest, Romania \\ * Correspondence: alecsandru.chirosca@ccpr.ro
}

Received: 27 April 2020; Accepted: 23 June 2020; Published: 26 June 2020

check for updates

\begin{abstract}
An adequate simulation model has been used for the calculation of angular and energy distributions of electrons, protons, and photons emitted during a high-power laser, 5- $\mu \mathrm{m}$ thick Ag target interaction. Their energy spectra and fluencies have been calculated between 0 and 360 degrees around the interaction point with a step angle of five degrees. Thus, the contribution of each ionizing species to the total fluency value has been established. Considering the geometry of the experimental set-up, a map of the radiation dose inside the target vacuum chamber has been simulated, using the Geant 4 General Particle Source code, and further compared with the experimental one. Maximum values of the measured dose of the order of tens of $\mathrm{mGy}$ per laser shot have been obtained in the direction normal to the target at about $30 \mathrm{~cm}$ from the interaction point.
\end{abstract}

Keywords: spectra of laser accelerated particle beams; mapping of radiation dose; GEANT4 simulations

\section{Introduction}

High-intensity lasers have progressively been used in contemporary research for the study of matter under extreme conditions and to generate beams of accelerated particles [1-6].

As result of the interaction of high-power laser pulses (I $>10^{19} \mathrm{~W} / \mathrm{cm}^{2}, f_{S}$ to $p s$ pulse duration) with solid micrometer flat $[1,2]$ or structured thin targets $[2,6]$, by the target normal sheath acceleration (TNSA) mechanism, electron and proton beams with high directionality, small divergence, and energies up to tens of $\mathrm{MeV}$ [1-6] are generated.

The TNSA regime involves complex physical phenomena and is usually considered to be the main rear surface ion acceleration mechanism. When the laser pre-pulse interacts with the target's front side, 
it produces a pre-plasma. The subsequent arrival of the main laser pulse leads to the generation of hot electrons, as the pre-plasma electrons absorb a percentage of laser pulse energy. The mean free path of such hot electrons in the target is larger than its thickness, and thus part of them pass through the target and form a dense sheath of negative charge in the proximity of the target rear surface up to its rear surface, where it generates a dense sheath. The further expansion of the electrons sheath into the vacuum determine a TV/m electric field, normal to the target surface. The impurities (water and organic molecules) adsorbed on the rear side of the target can be ionized in this strong electric field. Thus, the generated protons are accelerated in the normal direction to the target.

Besides electron and proton beams, bremsstrahlung radiation is also produced due to electron interaction with target nuclei [2]. At the same time, $\mathrm{X}$-ray bremsstrahlung photons can be provided by the "hot" electrons and laser accelerated electrons which reach the vacuum chamber walls $[7,8]$.

The mixed field of photons and electrons might create a hazardous radiation environment, as dose levels of tens of mGy/per laser shot, depending on the target thickness and material characteristics, can be obtained $[7,8]$.

In order to distinguish between different kinds of laser accelerated particle beams, complex detection systems needed to be envisaged. For such goals, magnetic or Thomson parabola spectrometers coupled with Lanex foils, image plates, radiochromic films (RCF), microchannel plates, or CR-39 detectors have been used [2,9-11]. These spectrometers, placed at different distances and angles with respect to the laser-target interaction point, can reveal the energy spectral distribution of the electron and proton beams. The spectra of photons emitted in TNSA regime can be measured using $X$-ray spectrometers and estimated from the energy distribution of electron beams [12]. The charts of electron-photon distribution in the mixed field of radiation were calculated and experimentally determined $[7,8]$.

It was shown [5] that the spectral distribution of laser accelerated particle beams in high power laser-solid target experiments can reproduce the space radiation environment. The values of radiation dose per laser shot measured during such experiments, inside the target vacuum chambers $[7,8]$, are similar to those measured onboard space vessels [13-18]. On the NASA website [13], daily values of hundreds of $\mu \mathrm{Gy}$ were reported for the cumulative radiation dose inside spacecrafts. Similar conditions can be obtained using laser plasma accelerator facilities available worldwide [19-23].

Inside the spacecrafts and space stations there are lots of electronic systems that need to be tested in dedicated facilities on Earth, before being used in extreme conditions. In addition, the assessment of detectors' response in ground-based facilities is essential for overcoming the problems intrinsic to space dosimetry. As shown in Hidding's et al. paper [5], to test electronics, a proper knowledge of the fluxes of ionizing radiation is an important issue.

In this context, the measurement and estimation of the spatial and angular spectral distribution of the ionizing radiation generated in high power laser-thin solid target experiments can be extremely useful.

In this paper, we propose a simulation model to be used for the assessment of the radiation dose map in a TNSA regime for a high power laser-thin solid target interaction experiment. First, the energy spectral distribution of electron, proton and photon beams, have been calculated within $0-360^{\circ}$ for a $5 \mu \mathrm{m}$ thick Ag target irradiated with a high-power laser pulse of about $5 \times 10^{19} \mathrm{~W} / \mathrm{cm}^{2}$ intensity. To this goal, the TNSA plasma expansion model has been considered in conjunction with the Geant4 Monte-Carlo radiation transport code. Then, the angular and energy spectral distributions of ionizing particles were used as input data for the calculation of electron, proton, and photon fluencies around the high power laser-thin solid target interaction point.

The values of input parameters are characteristics to a high power laser-thin solid target experiment performed at the CETAL laser facility [24].

In the Groza's et al. paper [24], we reported a method for the assessment of the energy of the accelerated proton beams produced in high power laser-thin solid target experiments using a stack of CR-39 detectors. It was also stated that a compact experimental set-up which connect the target 
holder with the detector holder, and the laser spot optical analysis system can be useful for practical applications. For example, by using such a system, the misalignments between targets and detectors can be avoided [24].

In this paper, we introduced an updated version of the above-mentioned experimental set-up which is also suitable for estimation of laser-accelerated electron beam energies. It includes a magnetic spectrometer and a cylindrical holder for positioning the EBT3 RCF passive detectors. The calibration of the RCF was performed for both X-ray photon beams and monoenergetic electrons.

The radiation dose map measured inside the interaction chamber during the high power laser-Ag thin target interaction experiments using EBT3 RCF detectors, will be presented. It will be compared with the simulated one, generated by implementing the data characteristic to angular and energy spectral distribution of electrons, protons, and photons in the geometry mesh of the experimental set-up designed using the GEANT4 standard geometry components.

\section{Description of the Simulation Models and Experimental Set up}

2.1. Description of the Model for Generation of Angular Spectra of Electrons, Protons and Photons; GEANT4 Model for Compute the Dose Map inside the Interaction Chamber

\section{(a) Description of TNSA Model}

The simulation model is based upon the Geant4 [25] framework. The mathematical models for sources description and data processing pipelines have been implemented in the Python language [26]. The integration between the sources description and the custom GEANT4 based application developed in $\mathrm{C}++$, was performed using the Geant 4 General Particle source. We used the command line scoring system for the region of interest and the output data were analyzed using another Python pipeline. The particle sources were implemented considering the target normal sheath acceleration (TNSA) mechanism and the plasma expansion model [1].

In TNSA regime, the interaction of a high power laser beam with a thin target generates on its front surface energetic electrons, which are accelerated in forward direction through the target. During their interaction with the target material, these hot electrons can generate bremsstrahlung radiation $[7,8]$. The electrons, which attain the back of the target and enter in vacuum, create an electrostatic space charge sheath and thus an electric field with an intensity of about $10^{12} \mathrm{~V} / \mathrm{m}$. Due to this field, the protons from the rear target surface can be accelerated up to $\mathrm{MeV}$ energies [1-6]. The temperature of hot electrons, is related to the laser intensity, [1]:

$$
T_{\text {hot }}=\left[\left(1+I\left[\mathrm{~W} / \mathrm{cm}^{2}\right] \lambda^{2}[\mu \mathrm{m}] /\left(1.37 \times 10^{18}\right)\right]^{1 / 2}-1\right) m_{0} \mathrm{c}^{2},
$$

where $I$ represent the laser intensity in beam focus, $\lambda$ is laser wavelength, $m_{0}$ electron mass, and c light velocity [1]. The number of electrons accelerated into the target, $N_{e}$, depends on the energy fraction absorbed by hot electrons $f=1.2 \times 10^{-15} I^{0.74}\left(\mathrm{~W} / \mathrm{cm}^{2}\right)$, through the formula [1,27]:

$$
N_{e}=f E_{L} / T_{h o t}
$$

where $E_{L}$ is the laser energy.

The electron density at the rear side of the target is $n_{e, 0}=N_{e} /\left(c \tau_{L} S_{\text {sheath }}\right)$, where $S_{\text {sheath }}=\pi\left(r_{0}+d \times\right.$ $\tan \theta)^{2}[1], \tau_{L}$ is laser pulse duration, $d$ is the target thickness, $r_{0}$ is the radius of laser beam in focus and $\theta$ is the half angular broadening of the hot electrons inside the target. Roth calculated this formula as a function of target thickness and laser intensity [27]:

$$
\begin{gathered}
n_{e, 0}=\left[\eta E_{L}\right] /\left[c \tau_{L} \pi\left(r_{0}+(d \tan \theta / 2)\right)^{2} k_{B} T_{h o t} \sim 1.5 \times 10^{19}\left[r_{0}^{2} /\left(r_{0}+(d \tan \theta / 2)^{2}\right]\left[I_{18} 7 / 4 /[(1+\right.\right.\right. \\
\left.\left.0.73 I_{18} \lambda\right)^{1 / 2}-1\right]\left[\mathrm{cm}^{-3}\right]
\end{gathered}
$$

where $I_{18}$ is the laser intensity in terms of $10^{18} \mathrm{~W} / \mathrm{cm}^{2}$ [27]. 
The energy spectrum of electrons leaving the rear side of the target is given by different formulas, depending on how large the laser intensity is:

$$
d N / d E \sim E^{2} e^{-E / T} \text { hot }
$$

(relativistic Maxwelian distribution) for $I>10^{19} \mathrm{~W} / \mathrm{cm}^{2}[7,12]$ and

$$
d N / d E \sim E^{1 / 2} e^{-E / T} \text { hot }
$$

(Maxwelian distribution) for $I<10^{19} \mathrm{~W} / \mathrm{cm}^{2}[7,12]$.

The energy spectrum of accelerated protons within the plasma expansion model is [1]:

$$
d N / d E=\left[n_{e, 0} c_{s} t_{\text {acc }} S_{\text {sheat }} /\left(2 E T_{\text {hot }}\right)^{1 / 2}\right] \exp \left(-\left(2 E / T_{\text {hot }}\right)^{1 / 2}\right), \text { where } c_{s}=\left(Z_{i} \times T_{\text {hot }} / m_{i}\right)^{1 / 2}
$$

where $t_{a c c}=1.3 \times \tau_{L}$ while $m_{i}$ and $Z_{i}\left(Z_{i}=1\right)$ are referred to as protons.

The energy spectrum of bremsstrahlung photons is derived from the energy spectrum of the electrons which interact with the Ag target $(Z=49)$ and was calculated considering the temperature of hot electrons. The bremsstrahlung radiation is due to energy deposited by the hot electrons while passing through the target.

(b) Description of Electron and Proton Sources Used for Calculation of Electron, Proton and Photon Spectra and Fluencies, around the High Power Laser-Thin Solid Target Interaction Point

The simulation model used for calculation of angular distribution of laser driven accelerated particles was developed considering mainly the above formulas (Equations (1)-(6)). A $2 \mathrm{MeV}$ temperature for the hot electrons was calculated using Formula (1) and the following experimental parameters [24]: $5 \times 10^{19} \mathrm{~W} / \mathrm{cm}^{2}$ laser intensity, 40 fs laser pulse duration, $207 \mu \mathrm{m}^{2}$ laser spot area.

Two volume sources of radiation were considered in order to compute the spectra of electrons, protons and photons emitted in the $0-360^{\circ}$ range (with a 5-degree angular step) at $3 \mathrm{~cm}$ distance, around the interaction point.

The first source represents the source of electrons (a disk of $9 \mu \mathrm{m}$ radius) generated in the pre-plasma obtained after the interaction of the PW laser with the target surface. It provides the electrons for the simulation of radiation transport. The electrons were considered to have a Maxwellian energy distribution in accordance with Equations (4) and (5). This source is position in front of the target [28-31]. The energy distribution of electrons was calculated, based on mathematical equations from (1) to (5). The angular distribution of electron source was assumed isotropic. The electrons generated in the pre-plasma which emit in $4 \pi$ are the main source for radiation in the vacuum chamber $[7,8]$.

The energy distribution of photons has been calculated considering the hot electrons interaction with the target nuclei.

The second source (a disk of 9- $\mu \mathrm{m}$ radius) of radiation included in the simulation was placed behind the target and provides the accelerated protons. There are considered the TNSA mechanism (Equations (1)-(3) and (6)) and the plasma expansion model [1,28,29]. The divergence of this source is an independent parameter and is defined through its half angle which was $25^{\circ}$ in accordance with [1].

The incidence angle of the laser beam on the target was not considered in these simulations. Previously, in the paper of Morita's et al. [32] by Particle in Cell simulations it was shown that an oblique incidence angle of laser on the target increases the energy of accelerated protons without changing the distribution of electrons. At laser intensities of $\sim 10^{19} \mathrm{~W} / \mathrm{cm}^{2}$, the incidence angle of the laser beam on the target influences only the efficiency of the energy transfer from photons to electrons into the pre-plasma.

The characteristics of source terms are summarized in Table 1. 
Table 1. Characteristics of source terms.

\begin{tabular}{ccc}
\hline Source of Radiation & Electrons (Source 1) & Protons (Source 2) \\
\hline Laser intensity $\left(\mathrm{W} / \mathrm{cm}^{2}\right)$ & $5 \times 10^{19}$ & $5 \times 10^{19}$ \\
Laser pulse duration $(\mathrm{fs})$ & 40 & 40 \\
Laser spot area $\left(\mu \mathrm{m}^{2}\right)$ & 207 & 207 \\
Source radius $(\mu \mathrm{m})$ & 9 & 9 \\
Source position & ahead & behind \\
Angular distribution & $4 \pi$ & $25^{\circ}$ half angle \\
Number of events & $1.97 \times 10^{12}$ & $1.29 \times 10^{9}$ \\
\hline
\end{tabular}

The two volume sources described above were used to compute the angular and energy spectral distributions of electrons, protons and photons around the high power laser-thin solid target interaction point using the GEANT4 simulation toolkit $[25,33,34]$. Particle fluencies were also determined.

The general particle sources (GPS) defined in GEANT4 provide a complex environment which allows the inclusion into the simulation of both proton and electron sources, with a specific ratio of particle numbers. It was considered a $1.97 \times 10^{12}$ total events number, $\left(1.97 \times 10^{12}\right.$ electron events number and $1.29 \times 10^{9}$ proton events number) and a proton to electron number ratio of $1.53 \times 10^{3}$. The total events number and the ratio between electron and proton events number were generated by analytical calculations using the TNSA model (Equations (1)-(6)) for the experimental parameters mentioned above.

Geant4 simulations were performed using the G4EmLivermore interaction library for electromagnetic radiation and QGSP_BIC_HP for proton interaction. Binary cascade models were activated for Ion interactions [33,34].

Monte Carlo algorithms provide a statistical approach for radiation transport within environments and detectors, the uncertainties being lower than $1 \%$ for simulations that runs for $10^{12}$ events number. The main source of inconsistencies in Monte Carlo simulations are represented by the variance reduction techniques [35] usually employed for decreasing the simulation run time. Such method was not applied here.

Scoring was performed using the Geant4 command line-based scoring system and a cylindrical mesh. The scoring mesh was binned in polar coordinates and had 5 degree angular and $0.2 \mathrm{~cm}$ radial steps, respectively. Proton, electron, and photon spectra, as well as their corresponding fluencies were assessed.

The simulations were performed using a 32 core XENON E5-2650 with 20 GB RAM. The run time was approximately of $70 \pm 4 \mathrm{~h}$ per simulation and the statistical fluctuations were less than $1 \%$.

The data were made available in a familiar web interface using Jupyter Notebooks [36]. This approach allowed for interactive data processing techniques to be applied to validate the simulation results. Thus, the output data files were easily integrated into other software packages for data analysis and further processing.

(c) Description of the Geometry Mesh of the Experimental Set-Up Used for Calculation of Radiation Dose Map Inside the Vacuum Chamber

The simulation of radiation dose map was also performed with the Geant4 v.10.5 framework tools described above $[25,33,34]$.

Firstly, the electron and proton sources were implemented into a mesh (see Figure 1) adapted to the specific parameters of the experimental set-up presented in Figure 2. The electron source (the first source of radiation) is illustrated as a yellow disk, the red disk represent the $5 \mu \mathrm{m}$ thick target behind which is positioned the proton source (the second source of radiation) designed as a green disk. The two sources were integrated into the simulation model using the GPS that allows the positioning of more than one source within an experimental set-up. Also, it was considered, that, the electrons that propagate in vacuum can generate nuclear reactions when interact with experimental set-up 
components (see Figure 2), producing secondary radiation such photons. For the considered particles energy range, in the simulation model, all available nuclear processes (in GEANT4 General Particle Source tools) were considered. The mesh of the experimental set-up was implemented using GEANT4 standard geometry components without any Boolean operations, and the overlapping regions were tested at the beginning of each run.

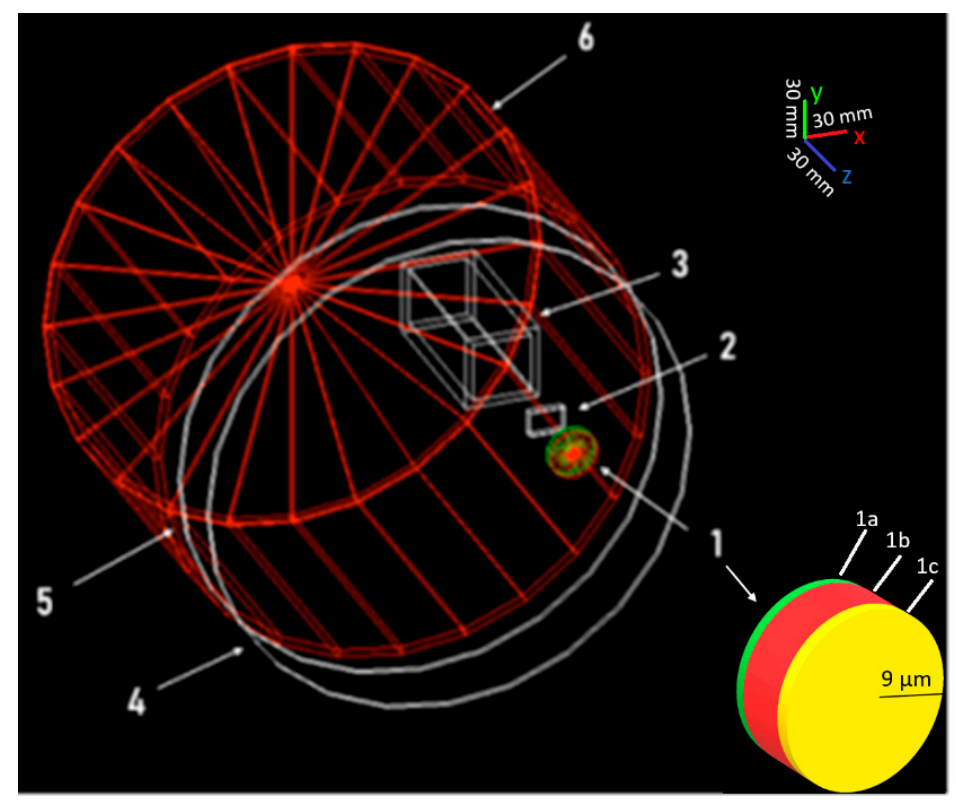

Figure 1. Geometry mesh of the GEANT4 simulation model: 1a-source of electrons; 1b-target; 1c-source of protons; 2-hole; 3-magnetic spectrometer; 4-target holder; 5-detector holder; 6-aluminum cylinder. The structure of the electron, proton sources and target are presented in the right corner of the image.

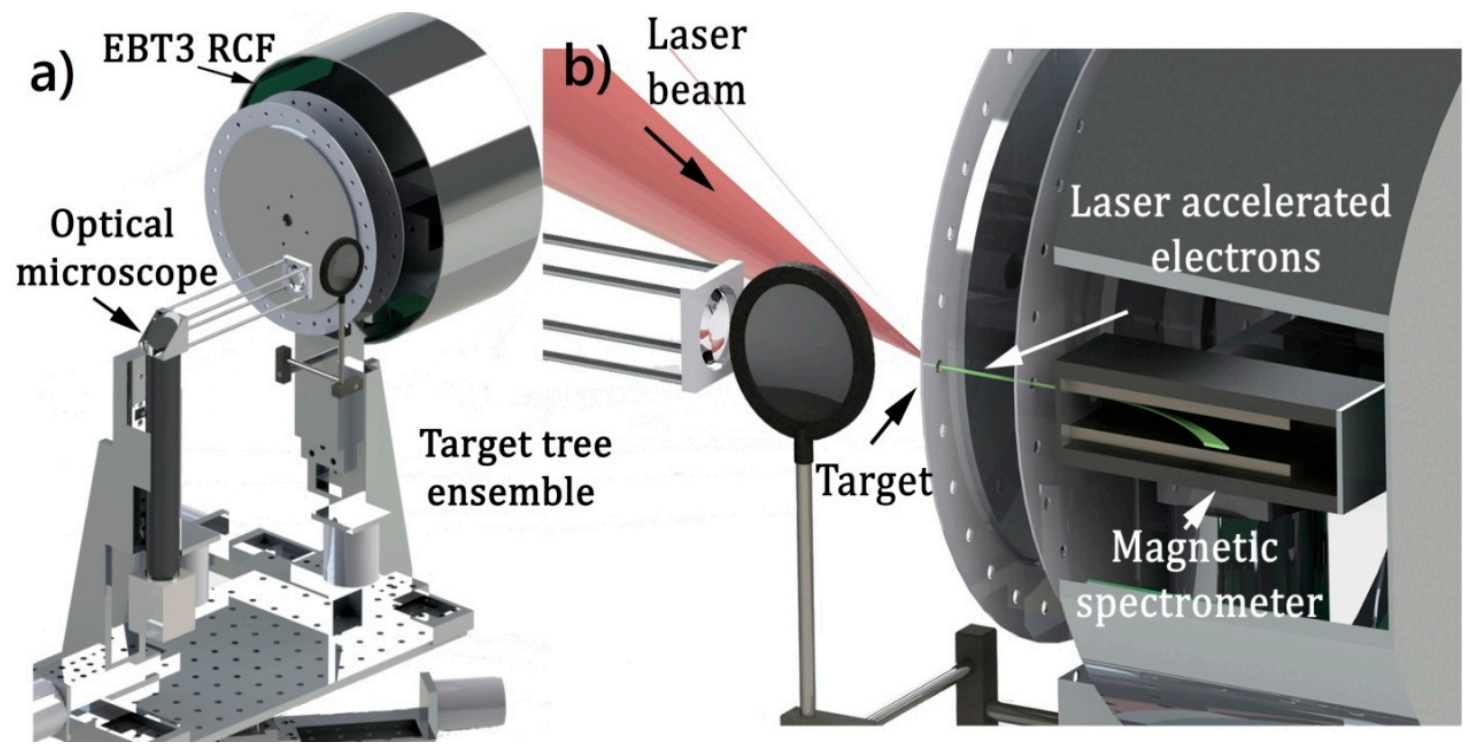

Figure 2. (a) Experimental set-up; (b) Detailed of the experimental set-up.

The angular and energy spectral distributions of electrons, protons and photons obtained as result of the interaction of laser spot with the Ag target, as well as the geometry mesh from Figure 1, were used to simulate the radiation dose map inside the vacuum chamber. 


\subsection{Experimental Setup Used to Measure Electron and Proton Spectra at $3 \mathrm{~cm}$ Distance from Target $n$}

The mapping of the radiation dose inside the target chamber has been performed during experiments [24] of high power-thin solid target interaction for a laser intensity in focus of about $5 \times 10^{19} \mathrm{~W} / \mathrm{cm}^{2}$ (40 fs pulse duration [24,37]). The target was tilted at $45^{\circ}$ with respect to the laser beam focus position. The laser spot surface area was $(9 \times 23) 207 \mu \mathrm{m}^{2}$ and the fraction of energy in the laser spot (at full width half maximum) was $\sim 30 \%$. The elliptical shape of the focal spot was previously attributed to the slightly ellipticity of the incoming beam $[8,38]$.

The experimental set-up from Figure 2, was designed to allow a straightforward analysis of laser accelerated electron and proton beams.

It is composed of the targets and passive detectors holder ensemble, a magnetic spectrometer $(\mathrm{B} \sim 0.6 \mathrm{~T})$, and an optical analysis ensemble. The target and detector holder system consist in two parallel metallic plates centered using a horizontal metallic ax. The targets were positioned on the first plate while the passive detectors used for proton beams analysis were placed on the second plate. The spectral analysis of the proton beams achieved using CR-39 detectors was reported in [24]. Behind the second plate a centered (to the drilled holes) magnetic spectrometer, for the estimation of the energy of the laser accelerated electrons, was placed in a fix position, while the target and detector holder ensemble can be rotated. Around the magnetic spectrometer can be observed a metallic cylinder that can be simultaneous rotated with the target and detector holder plates. On the inner surface of the cylinder (see Figure 2) EBT3 radiochromic films were positioned. A maximum electron energy of about $13 \mathrm{MeV}$ was estimated from the coloring of the EBT3 radiochromic films. Such values of the electron energies were also reported in similar experimental conditions [5].

The target and detector holder ensemble are placed on mechanical translation and rotation stages for precise alignment of each target in the focus of the laser beam. The optical analysis system is described in detail in [24].

\subsection{Calibration of EBT3 Radiochromic Films used for Mapping of the Radiation Field}

The dosimetry assessment during high power laser-thin solid target interaction performed using the EBT3 radiochromic films (RCF) proved to be useful [39] and offers two-dimensional information on the radiation dose. The EBT3 films have a symmetrical layer's structure as the active layer $(28 \mu \mathrm{m}$ thick) is sandwiched between two $125 \mu \mathrm{m}$ matte-polyester substrates (Ashland Advanced Materials). These polyester layers prevent the formation of Newton ring interference patterns when the irradiated EBT3 films are scanned with flatbed scanners [39].

The EBT3 films were calibrated using both high energy photons generated by a $40 \mathrm{kV}$ X-ray source [40] and electrons with an energy of about $5 \mathrm{MeV}$ using a classical medical accelerator. In the paper of Sorriaux et al. [41], it was shown that EBT3 RCF can be used for dosimetry measurements of photon, electron and proton beams as their calibration curves (in the same dose range), are similar. The uncertainties of the EBT3 calibration curves presented there were within 1.5\% for photons and protons and $2 \%$ for electrons [41].

A high precision measurement of the optical density of each EBT3 film is essential for the evaluation of the ionizing particles radiation dose values inside the target chamber.

The EBT3 films exposed during the high-power laser thin solid target experiments, as well as those used for the determination of the calibration curve (see Figure $3 \mathrm{~b}$ ) were scanned using an EPSON Expression 11000XL professional scanner with a resolution of $4800 \mathrm{dpi}$ in transmission mode. The scanned area was about $0.5 \times 0.5 \mathrm{~cm}^{2}$ for each film. 

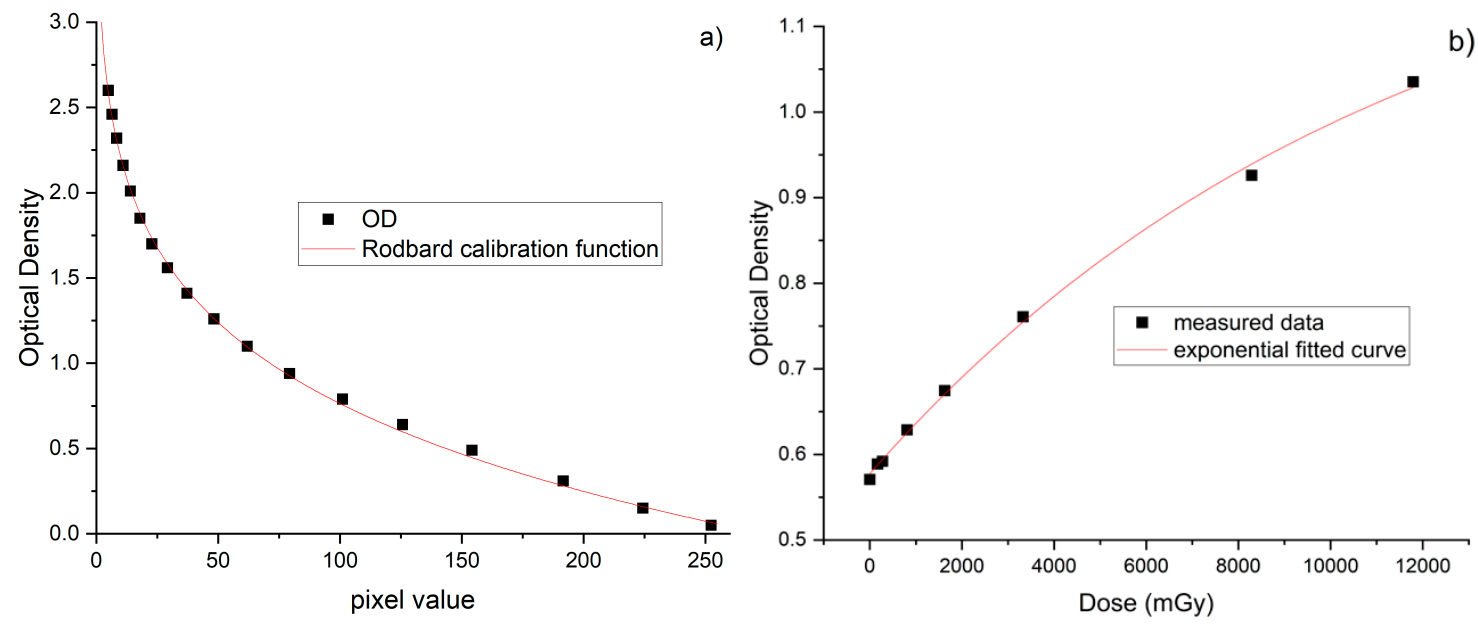

Figure 3. (a) Pixel values to optical density Rodbard calibration curve. (b) The dependence of optical density on radiation dose.

The scanned images of the EBT3 films were processed with ImageJ software in order to convert the pixel values into standard optical density (OD) [42]. For this purpose, a Kodak calibrated optical density step tablet [42] has been used and the images were converted into 8 bits grey scale. The pixel values range from $0-255$ gray units. A so-called Rodbard calibration function $[43,44]$ was used for pixel values to obtain an optical density transformation: $\mathrm{y}=d+(a-d) /\left(1+(\mathrm{x} / c)^{b}\right)$ where $a=80.147399$, $b=0.102551, c=1.109687 \times 10^{12}, d=8.304216$. The $\mathrm{R}^{2}$ was 0.997 . This function was identified to be suitable for pixel values to OD calibration in medical applications and for the establishment of dose response curves $[43,44]$. The graph of the Rodbard function is presented in Figure 3a.

The calibration graph presented in Figure $3 \mathrm{~b}$ was obtained for a $40 \mathrm{keV}$ photons beam. The calibration curve obtained for $5 \mathrm{MeV}$ electrons is similar (within $\pm 7 \%$ ) with that one from Figure $3 \mathrm{~b}$. Both curves were used for calculation of the radiation dose inside the target chamber as well as for the electron maximum energy (of about $13 \mathrm{MeV}$ ) estimation. The black squares represent the measured data values and in red is the curve used for fitting. As fitted function, we used an exponential one. The fitting parameters was: $R^{2}=0.997$, and standard error was about 0.069 .

\section{Results and Discussions}

(a) Simulation Results on the Angular Spectral Distribution of Electron, Proton and Photon Beams Generated in TNSA Regime

Laser accelerated electron and proton beams in vacuum are the primary sources of radiation in a high power laser-thin solid target interaction experiment in the TNSA regime. The secondary radiation source consists mainly of bremsstrahlung $\mathrm{X}$-ray photons or gamma rays both produced by the interaction of hot electrons with target nuclei. The interaction of primary radiation, (mainly of electrons) with the vacuum chamber walls and detectors placed at different distances and inclination angles with respect to the laser-target interaction point, also generate bremsstrahlung radiation on a nanosecond time scale.

The opening angle of proton and electron accelerated beams depend on few parameters such as: target thickness, laser intensity, laser focal spot size, emittance, and source dimensions [1-6]. Many authors [1-6,27,45-47] reported that electron and proton beams generated in TNSA regime have the highest number and energy in forward direction, normal to the target. The decrease of protons energy with the increase of opening angle was reported both by calculations and experimental measurements $[9,45]$.

The spectra of proton and electron accelerated beams as well as of the emitted photons calculated using the simulation model described above (without considering the mesh of the experimental set-up 
presented in Figure 1) allowed us to find angular and energy distributions of primary and secondary radiation (see Figures 4 and 5).
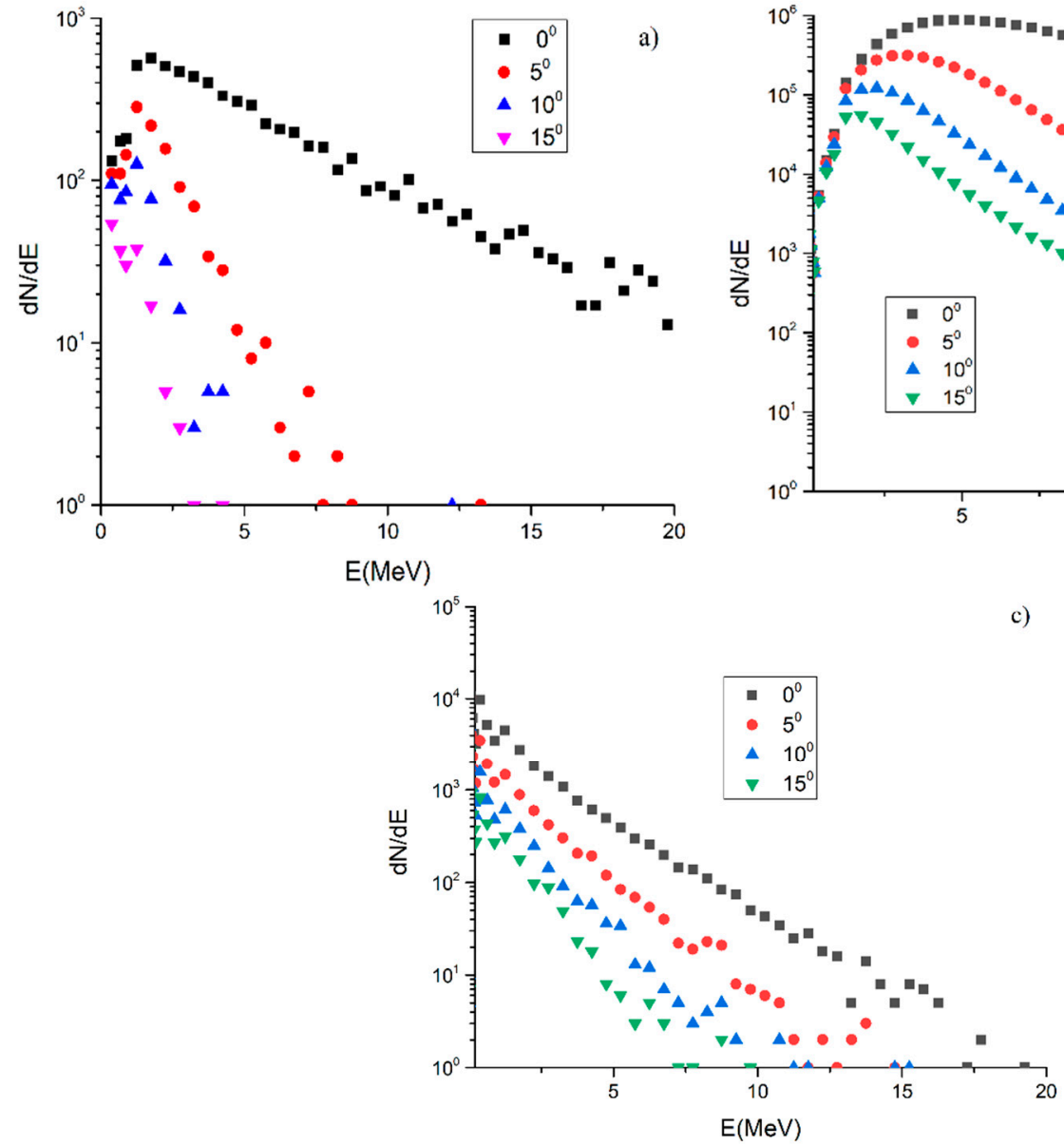

Figure 4. Simulated spectra of laser accelerated: (a) protons; (b) electrons beams and (c) emitted photons at different angles within $0-15$ degrees range. $0^{\circ}$ is considered in forward direction normal to target.
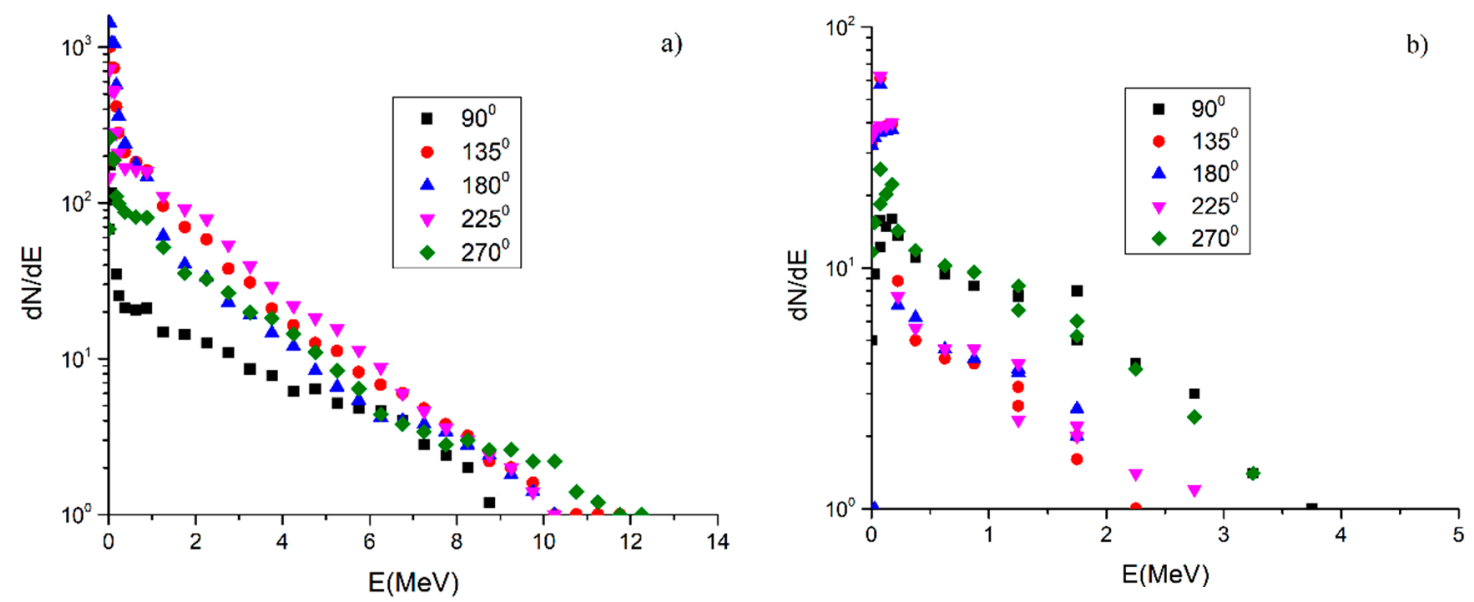

Figure 5. Simulated spectra of laser accelerated: (a) electron and (b) photon beams at different angles.

Within 0-15 degrees of angle, the spectra of electrons, protons, and photons are shown in Figure 4 $\left(0^{\circ}\right.$ is considered in forward direction normal to the target position). These spectra are calculated for the pulse duration time, $5 \times 10^{19} \mathrm{~W} / \mathrm{cm}^{2}$ laser intensity, and at $3 \mathrm{~cm}$ distance from the laser-target 
interaction point. In Figure 4a can be observed, that the most energetic protons are emitted in normal direction to the target position. Within 5 degrees angle the energy of protons drastically decreases. However, the electron spectra presented in Figure $4 \mathrm{~b}$ indicate only the decrease of electron numbers as the angle increases (see Figure $4 \mathrm{~b}$ ). The spectra of photons also display an angular dependence (see Figure 4c). The spectra obtained for angles between $345^{\circ}$ and $0^{\circ}$ degrees are similar.

The interaction between the laser focused spot and target was considered to produce ionizing radiation within $0-360^{\circ}$. The spectra of electrons and photons at different angles between $90^{\circ}$ and $270^{\circ}$ are presented in Figure 5. The number of emitted electrons and photons as well as their energy are lower than those emitted in the $0-15^{\circ}$ range (see Figure 4). Proton spectra were not generated in this angular range.

The spectra of electrons, protons, and photons (see Figures 4 and 5) display some fluctuations in the distribution of particles as function of energy. It appears mainly at the highest energies and at angles different from $0^{\circ}$. This is probably due to the statistical approach characteristic to the Monte Carlo simulations $[33,34]$ and to the fact that the number of generated particles at high energies, is low. Anyway, these fluctuations are relatively small and do not affect the estimations of the electron, proton, and photon spectral distributions. Similar distributions, but without any fluctuations, can be obtained when the spectra are analytically calculated. However, such calculations are possible only at $0^{\circ}$.

The main advantage of the constructed simulation model is that the simultaneous calculations of electron, proton and photon spectra and it angular dependence within $0-360^{\circ}$ can offer an overall image on the ionizing radiation distribution around the high power laser-thin solid target interaction point. Thus, we can evaluate the evolution of the number of produced electrons, protons, and photons both with energy and angle as a function of experimental parameters such as: laser intensity, laser pulse duration, and the target thickness.

(b) Simulation results on Electron, proton, and Photon Fluencies

High-power laser-thin solid target interaction generates mixed radiation fields of electrons and photons. The radiation dose measured inside the vacuum chamber by passive detectors, is mainly attributed to photons and laser accelerated electrons. The interaction of electrons with the metallic components of the chamber also generates bremsstrahlung radiation $[7,8]$. The protons are emitted in the target normal direction, forwards, and backwards [27], being stopped in materials in accordance to their stopping powers [48].

For near target radiation field assessment, the simulation model described above, allowed us the estimation of total fluencies of radiation generated by the interaction of the high power laser beam with the thin solid target. The fluencies of electrons, protons, and photons were also calculated (see Figure 6), using as input data the spectra presented in Figures 4 and 5. It turns out that electrons contribute $\sim 98.9 \%$, photons $\sim 1 \%$, and protons $\sim 0.1 \%$, respectively, to the total fluencies. The electrons, and photons are produced almost uniformly around the interaction point, having a maximum in the normal direction to the target position. The protons are emitted mainly in the forward direction. 

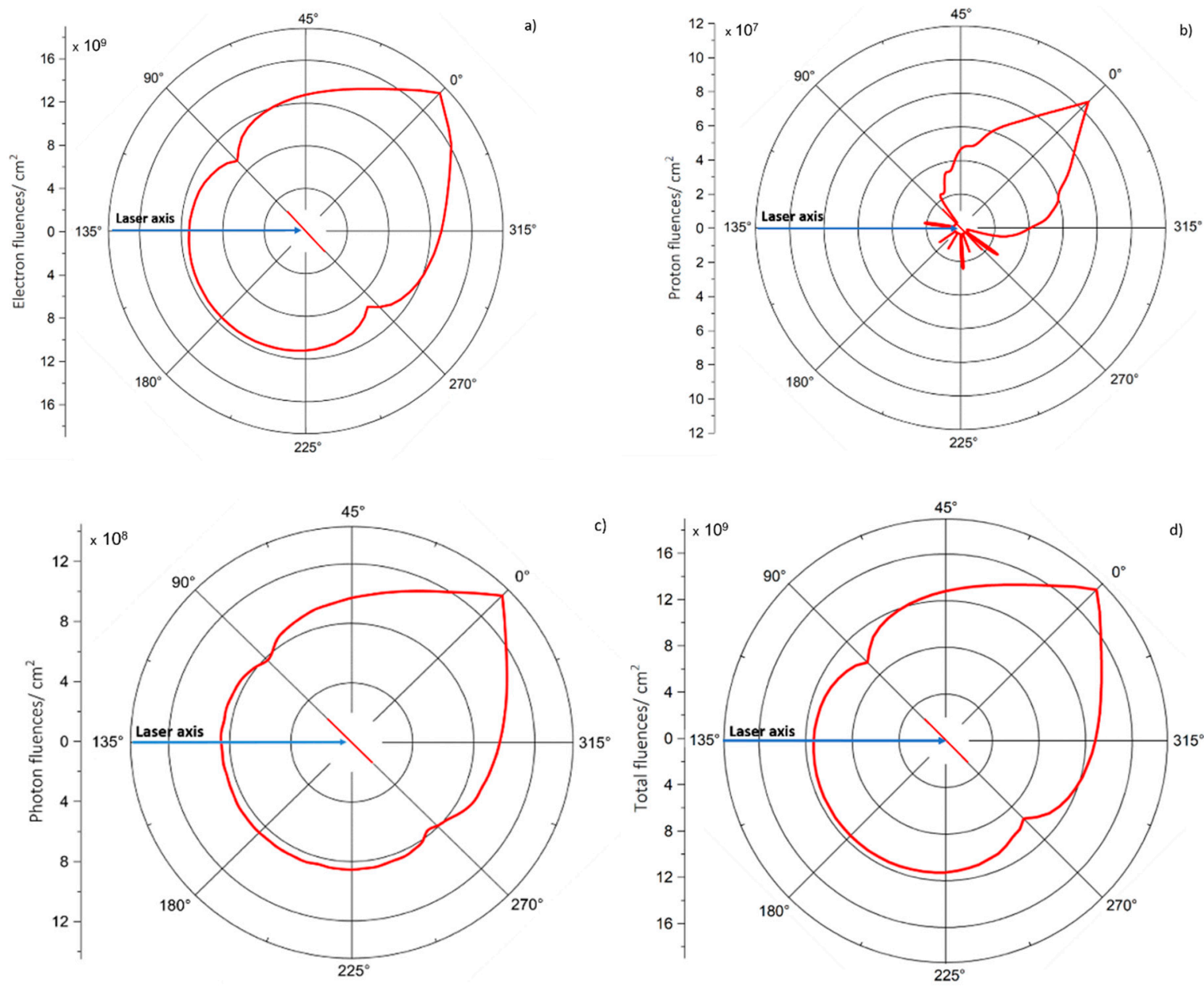

Figure 6. Angular dependence of particle fluencies $/ \mathrm{cm}^{2}$ at $3 \mathrm{~cm}$ from the interaction point on logarithmic scale: (a) electrons (b) protons (c) photons and (d) total fluencies.

\section{(c) Simulated and Experimental Dose Maps}

By implementing the angular and energy spectral distributions in the mesh associated with the experimental set-up displayed in Figure 2, the simulated dose map was generated.

In the experiments performed using the experimental set-up from Figure 2, the radiation dose inside the target chamber was charted, using EBT3 RCF placed at different distances and inclination angles with respect to the high power laser-thin solid target interaction point. The calibration curve from Figure $3 b$ was used for calculation of the radiation dose inside the vacuum chamber.

In Figure 7a, the radiation dose measured around the interaction point is shown, in comparison with the simulated one. The dose values are per laser shot and were normalized to $30 \mathrm{~cm}$ distance. As can be observed the maximum values of the radiation dose were reached in the forward target normal direction. 

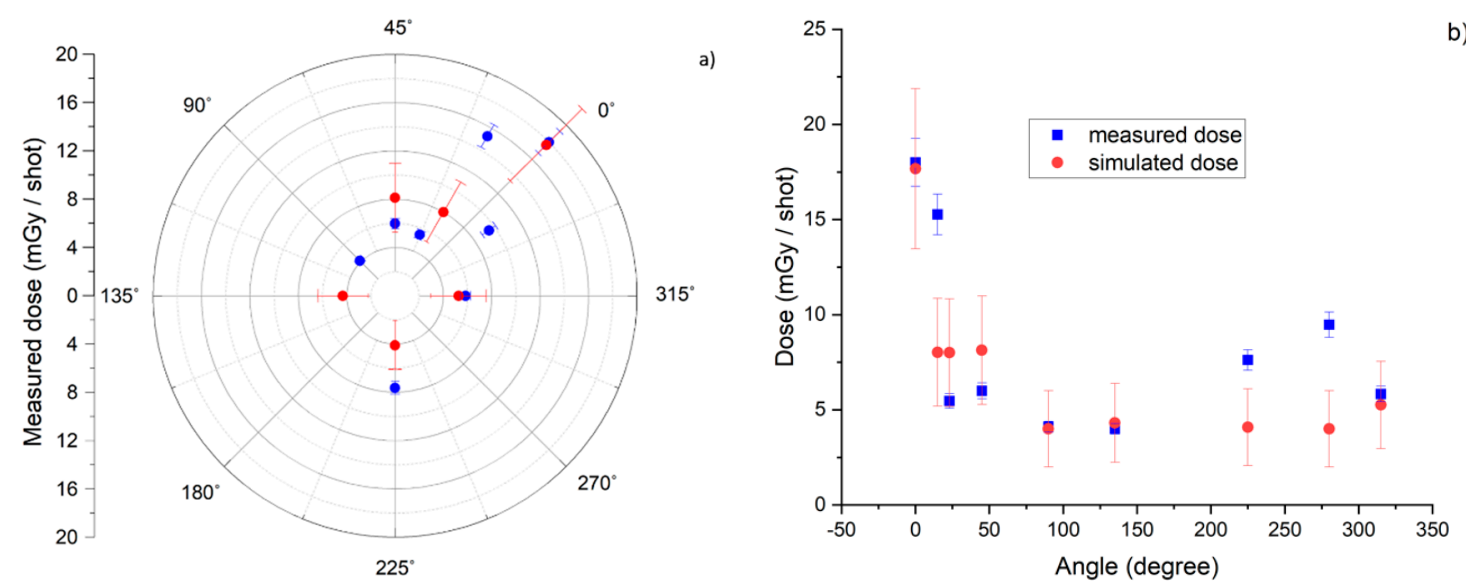

Figure 7. (a) Map of the measured dose (blue dots) inside the target chamber and of the simulated radiation dose (red dots); (b) The fluctuations in the angular distribution of measured and simulated dose.

There are certain inadequacies between experimental data and simulations (see Figure $7 \mathrm{~b}$ ). We suppose that these discrepancies appear as the scattering of the radiation on the walls of the vacuum chamber and from metallic cylinders placed around the magnetic spectrometer (see Figure 2), which was not considered in the simulation model. Otherwise, it would determine a percentage of uncertainties higher than $1 \%$ for $10^{12}$ events number, and the simulation run time would have increased accordingly. Therefore, a simplified geometry was considered.

Moreover, the vacuum chamber has a rectangular parallelepiped geometrical shape [20] and the maximum measured dose values away from the forward direction are in the proximity of the chamber corners. Measured dose values of tens of $\mathrm{mGy} /$ per laser shot were obtained in similar experimental conditions [8].

Considering the electron and photon fluency data presented in Figure 6, we suppose that the electrons have the largest contribution to the radiation dose (98.9\%) while photons contribute only $1 \%$. Protons do not influence the dose value by more than $0.1 \%$. Anyway, the protons with the highest energy are emitted in the forward direction (see Figure 6 and Ref. [27]), even if in the backward direction to the target position, they were also observed (see Figure 6 and Ref. [27]). The protons emitted forward are stopped in the detectors placed at $3 \mathrm{~cm}$ distance from the target position (see Figure 2).

Thus, based on the calculation of the angular dependence of particle fluencies, the radiation dose map reconstruction was performed in any point within the area of interest. The presented simulation model can be further developed for the calculation of dose in any material, by considering the energy and type of incident radiation as well as possible reactions.

\section{Conclusions}

The results presented in this paper link the angular and energy distributions of electrons, protons, and photons calculated within $0-360^{\circ}$, with an angular step of five degrees, to the radiation dose map. This is achieved through integration into the simulation model and the use of an experimental set up mesh. The geometry of the experimental set-up was designed in the Geant4 General Particle Source code and was considered for the calculation of the radiation dose around the high-power laser-solid thin foil interaction point. When comparing the calculated dose map with the experimental one, certain discrepancies between the values of dose obtained at different angles were observed. This is most probably because the scattering of radiation into the vacuum chamber was not considered in the simulation model. 
Integrated dose per laser shot of about $15 \mathrm{mGy}$ (due to electrons and photons) in the normal direction to the target, at $30 \mathrm{~cm}$ distance from the laser-target interaction point was experimentally measured.

Excepting the geometry of our experimental set-up, the presented simulation model allows for the calculation of the fluencies of electrons, protons, and photons based on their energy and angular spectral distributions. Our results show that electrons contribute almost $\sim 99 \%$ to the value of total fluencies generated around the interaction point, while photons have a $1 \%$ share.

The numerical and experimental investigations presented in this paper suggest that high power laser-thin solid target experiments can be used as a test environment for electronic devices placed onboard spacecraft and space stations, the main advantage being the simultaneous generation of electrons, protons, and photons.

Author Contributions: Conceptualization, A.G., A.C., B.B., and M.S.; methodology, A.G, B.B., M.S., E.S., and D.B.D.; investigation, B.B., M.S., E.S., and D.B.D.; software, A.C. and D.B.D.; validation, A.G., A.C., M.G., B.B., M.S., and E.S., D.B.D.; formal analysis, A.G. and A.C.; writing-original draft preparation A.G., A.C., E.S., B.B., E.S., M.S., D.B.D., and M.G.; writing-review and editing A.G., A.C., E.S., and D.B.D. All authors have read and agreed to the published version of the manuscript.

Funding: Work has been funded by European Space Agency within the ESA contract No. 4000121912/17/NL/CBi/2017.

Acknowledgments: We acknowledge support from the CETAL-PW facility at the National Institute for Laser, Plasma and Radiation Physics, Magurele, Romania. We also thank to Șerban Udrea from GSI-Darmstadt for fruitful discussions.

Conflicts of Interest: The authors declare no conflict of interest. The funders had no role in the design of the study; in the collection, analyses, or interpretation of data; in the writing of the manuscript, or in the decision to publish the results.

\section{References}

1. Fuchs, J.; Antici, P.; d'Humieres, E.; Lefebvre, E.; Borghesi, M.; Brambrink, E.; Cecchetti, C.A.; Kaluza, M.; Malka, V.; Manclossi, M.; et al. Laser-driven proton scaling laws and new paths towards energy increase. Nat. Phys. 2006, 2, 48-56. [CrossRef]

2. Macchi, A.; Borghesi, M.; Passoni, M. Ion acceleration by superintense laser-plasma interaction. Rev. Mod. Phys. 2013, 85, 751-793. [CrossRef]

3. Bolton, P.R.; Borghesi, M.; Brenner, C.; Carroll, D.C.; Martinis De, C.; Flacco, A.; Floquet, V.; Fuchs, J.; Gallegos, P.; Giove, D.; et al. Instrumentation for diagnostics and control of laser-accelerated proton (ion) beams. Phys. Med. 2014, 30, 255-270. [CrossRef] [PubMed]

4. Ledingham, K.W.D.; Galster, W. Laser-driven particle and photon beams and some applications. New J. Phys. 2010, 12, 045005. [CrossRef]

5. Hidding, B.; Karger, O.; Konigstein, T.; Pretzler, G.; Manahan, G.G.; McKenna, P.; Gray, R.; Wilson, R.; Wiggins, S.M.; Welsh, G.H.; et al. Laser-plasma-based Space Radiation Reproduction in the Laboratory. Sci. Rep. 2017, 7, 42354. [CrossRef]

6. Zigler, A.; Eisenman, S.; Botton, M.; Nahum, E.; Schleifer, E.; Baspaly, A.; Pomerantz, I.; Abicht, F.; Branzel, J.; Priebe, G.; et al. Enhanced Proton Acceleration by an Ultrashort Laser Interaction with Structured Dynamic Plasma Targets. Phys. Rev. Lett. 2013, 110, 215004. [CrossRef]

7. Liang, T.T.; Bauer, J.M.; Liu, J.C.; Rokni, S.H. Radiation protection around high-intensity laser interactions with solid targets. Health Phys. 2018, 115, 6. [CrossRef]

8. Liang, T.; Bauer, J.; Cimeno, M.; Ferrari, A.; Galtier, E.; Granados, E.; Lee, H.J.; Liu, J.; Nagler, B.; Prinz, A.; et al. Radiation dose measurements for high-intensity laser interactions with solid targets at SLAC, Rad. Prot. Dosim. 2016, 172, 346-355. [CrossRef]

9. Nurnberg, F.; Schollmeier, M.; Brambrink, E.; Blazevic, A.; Carroll, D.C.; Flippo, K.; Gautier, D.C.; Geißel, M.; Harres, K.; Hegelich, B.M.; et al. Radiochromic film imaging spectroscopy of laser accelerated proton beams. Rev. Sci. Instrum. 2009, 80, 033301. [CrossRef] 
10. Jeong, T.W.; Singh, P.K.; Scullion, C.; Ahmed, H.; Hadjisolomou, P.; Jeon, C.; Yun, H.; Kakolee, K.F.; Borghesi, M.; Ter-Avetisyan, S. CR-39 track detector for multi-MeV ion spectroscopy. Sci. Rep. 2017, 7, 2152. [CrossRef]

11. Jeong, T.W.; Singh, P.K.; Scullion, C.; Ahmed, H.; Kakolee, K.F.; Hadjisolomou, P.; Alejo, A.; Kar, S.; Borghesi, M.; Ter-Avetisyan, S. Experimental evaluation of the response of micro-channel plate detector to ions with 10s of MeV energies. Rev. Sci. Instrum. 2016, 87, 083301. [CrossRef] [PubMed]

12. Fiorini, F.; Neely, D.; Clarke, R.J.; Green, S. Characterization of laser-driven electron and photon beamsusing the Monte Carlo code FLUKA. Laser Part. Beams 2014, 32, 233-241. [CrossRef]

13. Zell, H. Space Weather. Text. NASA. Available online: http://www.nasa.gov/mission_pages/rbsp/science/ rbsp-spaceweather.html (accessed on 1 March 2020).

14. Tokumaru, M. Three-dimensional exploration of the solar wind using observations of interplanetary Scintillation. Proc. Jpn. Acad. Ser. B 2013, 89, 67-79. [CrossRef] [PubMed]

15. Cannon, P. Extreme Space Weather: Impacts on Engineered Systems and Infrastructures, in Royal Academy of Engineering. Available online: https://www.raeng.org.uk/publications/reports/space-weather-full-report (accessed on 5 March 2020).

16. Delzanno, G.L.; Borovsky, J.E.; Thomsen, M.F.; Moulton, J.D.; Macdonald, E.A. Future beam experiments in the magnetosphere with plasma contactors: How do we get the charge off the spacecraft? J. Geophys. Res. Space Phys. 2015, 120, 3647-3664. [CrossRef]

17. Holly, Z. National Aeronautics and Space Administration. Available online: https://www.nasa.gov/mission pages/rbsp/science/rbsp-spaceweather-human.html (accessed on 4 August 2017).

18. Konigstein, T.; Karger, O.; Pretzler, G.; Rosenzweig, J.B.; Hidding, B. Design considerations for the use of laser-plasma accelerators for advanced space radiation studies. J. Plasma Phys. 2012, 78, 383-391. [CrossRef]

19. Danson, C.; Hillier, D.; Hopps, N.; Neely, D. Petawatt class lasers worldwide. High Power Laser Sci. Eng. 2015, 3, e3. [CrossRef]

20. Center for Advanced Laser Technologies (CETAL), Ultra-intense Lasers Laboratory. Available online: http://cetal.inflpr.ro/newsite/cetal-pw (accessed on 20 February 2020).

21. Asavei, T.; Tomut, M.; Bobeica, M.; Aogaki, S.; Cernaianu, M.O.; Ganciu, M.; Kar, S.; Manda, G.; Mocanu, N.; Neagu, L.; et al. Materials in extreme environments for energy, accelerators and space applications at ELI-NP. Rom. Rep. Phys. 2016, 68, S275-S347.

22. Ganciu, M.; Groza, A.; Cramariuc, O.; Mihalcea, B.; Serbanescu, M.; Stancu, E.; Surmeian, A.; Butoi, B.; Dreghici, D.; Chirosca, A.; et al. Hardware and software methods for radiation resistance rising of the critical infrastructures. Rom. Cyber Secur. J. 2019, 1, 3-13.

23. Narici, L.; Casolino, M.; Fino, L.; Di Larosa, M.; Picozza, P.; Rizzo, A.; Zaconte, V. Performances of Kevlar and Polyethylene as radiation shielding on-board the International Space Station in high latitude radiation environment. Sci. Rep. 2017, 7, 1-11. [CrossRef]

24. Groza, A.; Serbanescu, M.; Butoi, B.; Stancu, E.; Straticiuc, M.; Burducea, I.; Balan, A.; Chirosca, A.; Mihalcea, B.; Ganciu, M. Advances in Spectral Distribution Assessment of Laser Accelerated Protons using Multilayer CR-39 Detectors. Appl. Sci. 2019, 9, 2052. [CrossRef]

25. Allison, J.; Amako, K.; Apostolakis, J.; Arce, P.; Asai, M.; Aso, T.; Bagli, E.; Bagulya, A.; Banerjee, S.; Barrand, G.J.N.I.; et al. Recent developments in Geant4. Nucl. Instrum. Methods Phys. Res. A 2016, 835, 186-225. [CrossRef]

26. Python Core Team. Python: A Dynamic, Open Source Programming Language; Python Software Foundation, 2015. Available online: https://www.python.org/ (accessed on 20 May 2020).

27. Roth, M.; Schollmeier, M. Ion Acceleration-Target Normal Sheath Acceleration. In Proceedings of the CAS-CERN Accelerator School: Plasma Wake Acceleration Geneva, Geneva, Switzerland, 23-29 November 2014.

28. Mora, P. Plasma expansion in vacuum. Phys. Rev. Lett. 2003, 90, 185002. [CrossRef] [PubMed]

29. Tampo, M.; Awano, S.; Bolton, P.R.; Kondo, K.; Mima, K.; Mori, Y.; Kodama, R. Correlation between laser accelerated $\mathrm{MeV}$ proton and electron beams using simple fluid model for target normal sheath acceleration. Phys. Plasmas 2010, 17, 7. [CrossRef]

30. Xiao, K.D.; Zhou, C.T.; Jiang, K.; Yang, Y.C.; Li, R.; Zhang, H.; He, X.T. Multidimensional effects on proton acceleration using high-power intense laser pulses. Phys. Plasmas 2018, 25, 2. [CrossRef] 
31. Volpe, L.; Fedosejevs, R.; Gatti, G.; Pérez-Hernández, J.A.; Méndez, C.; Apiñaniz, J.; Roso, L. Generation of high energy laser-driven electron and proton sources with the 200 TW system VEGA 2 at the Centro de Laseres Pulsados. High Power Laser Sci. Eng. 2019, 7, 6-11. [CrossRef]

32. Morita, T.; Esirkepov, T.Z.; Bulanov, S.V.; Koga, J.; Yamagiwa, M. Tunable High-Energy Ion Source via Oblique Laser Pulse Incident on a Double-Layer Target. Phys. Rev. Lett. 2008, 100, 145001. [CrossRef]

33. Agostinelli, S.; Allison, J.; Amako, K.A.; Apostolakis, J.; Araujo, H.; Arce, P.; Asai, M.; Axen, D.; Banerjee, S.; Barrand, G.; et al. Geant4-a simulation toolkit. Nucl. Instrum. Methods Phys. Res. A 2003, 506, 250-303. [CrossRef]

34. Allison, J. Geant4 developments and applications. IEEE Trans. Nucl. Sci. 2006, 53, 270-278. [CrossRef]

35. Kong, R.; Ambrose, M.; Spanier, J. Efficient, Automated Monte Carlo Methods for Radiation Transport. J. Comput. Phys. 2008, 227, 9463-9476. [CrossRef]

36. The Jupyter Notebook. Available online: https://jupyter.org/ (accessed on 21 May 2020).

37. Giubega, G. Proton Acceleration in Ultra-Intense Laser Interaction with Solid Targets at CETAL-PW Laser, WORKSHOP CETAL 2018. Available online: http://cetal.inflpr.ro/newsite/workshop_abstracts.php (accessed on 15 December 2019).

38. Zeil, K.; Kraft, S.D.; Bock, S.; Bussmann, M.; Cowan, T.E.; Kluge, T.; Metzkes, J.; Richter, T.; Sauerbrey, R.; Schramm, U. The scaling of proton energies in ultrashort pulse laser plasma acceleration. New J. Phys. 2010, 12, 045015. [CrossRef]

39. Najafi, M.; Geraily, G.; Shirazi, A.; Esfahani, M.; Teimouri, J. Analysis of Gafchromic EBT3 film calibration irradiated with gamma rays from different systems: Gamma Knife and Cobalt-60 unit. Med. Dosim. 2017, 3, 159-168. [CrossRef] [PubMed]

40. Available online: http://tomography.inflpr.ro/ (accessed on 15 March 2020).

41. Sorriaux, J.; Kacperek, A.; Rossomme, S.; Lee, J.A.; Bertrand, D.; Vynckier, S.; Sterpin, E. Evaluation of Gafchromic EBT3 films characteristics in therapy photon, electron and proton beams. Phys. Med. 2013, 6, 599-606. [CrossRef] [PubMed]

42. Available online: https://imagej.nih.gov/ij/docs/examples/calibration/ (accessed on 30 January 2020).

43. Available online: https://imagej.nih.gov/ij/docs/guide/146-30.html\#sub:Curve-Fitting (accessed on 30 January 2020).

44. DeLean, A.; Munson, P.J.; Rodbard, D. Simultaneous analysis of families of sigmoidal curves: Application to bioassay, radioligand assay, and physiological dose-response curves. Am. J. Physiol. 1978, 235, E97-E102. [CrossRef] [PubMed]

45. Hatchett, S.P.; Brown, C.G.; Cowan, T.E.; Henry, E.A.; Johnson, J.S.; Key, M.H.; Koch, J.A.; Langdon, A.B.; Lasinski, B.F.; Lee, R.W.; et al. Electron, photon, and ion beams from the relativistic interaction of Petawatt laser pulses with solid targets. Phys. Plasmas 2000, 7, 5. [CrossRef]

46. Cowan, T.E.; Fuchs, J.; Ruhl, H.; Kemp, A.; Audebert, P.; Roth, M.; Stephens, R.; Barton, I.; Blazevic, A.; Brambrink, E.; et al. Renard-Le Galloudec, N.; Ultralow Emittance, Multi-MeV Proton Beams from a Laser Virtual-Cathode Plasma Accelerator. Phys. Rev. Lett. 2004, 92, 20. [CrossRef]

47. Carroll, D.C.; McKenna, P.; Lundh, O.; Lindau, F.; Wahlström, C.G.; Bandyopadhyay, S.; Pepler, D.; Neely, D.; Kar, S.; Simpson, P.T.; et al. Active manipulation of the spatial energy distribution of laser-accelerated proton beams. Phys. Rev. E 2007, 76, 065401R. [CrossRef]

48. Ziegler, J.F.; Biersack, J.P. The Stopping and Range of Ions in Matter; Bromley, D., Ed.; Treatise on Heavy-Ion Science, Volume 6: Astrophysics, Chemistry, and Condensed Matter; Springer: Boston, MA, USA, 1985; pp. 93-129.

(C) 2020 by the authors. Licensee MDPI, Basel, Switzerland. This article is an open access article distributed under the terms and conditions of the Creative Commons Attribution (CC BY) license (http://creativecommons.org/licenses/by/4.0/). 